\title{
THE HARDY CLASS OF A FUNCTION WITH SLOWLY-GROWING AREA
}

\author{
LOWELL J. HANSEN
}

ABSTRACT. In this paper we show that if $f$ is analytic on the open unit disk and if the area of $[\{|z| \leq R\} \cap$ image of $f]$ grows sufficiently slowly as a function of $R$, then $f$ belongs to the Hardy class $H^{p}$ for all $p$ satisfying $0<p<+\infty$.

Let $\Delta$ denote the open unit disk $\{|z|<1\}$. It has been shown recently by H. Alexander, B. A. Taylor and J. L. Ullman [1, Theorem 1, p. 335] that

(1) if $f$ is analytic on $\Delta$ and the area of $f(\Delta)$ is finite, then $f$ belongs to the Hardy class $\mathrm{H}^{2}$; and

(2) if in addition $f(0)=0$, then $1 / 2\|f\|_{2}^{2} \leq$ Area of $f(\Delta)$.

In this note we prove a theorem which strengthens (1) above:

Theorem. Let $f$ be analytic on $\Delta$. For $R>0$, let

$$
A(R)=\text { Area of }[\{|z| \leq R\} \cap f(\Delta)] .
$$

If $A(R)\left(R^{-2} \log R\right) \rightarrow 0$ as $R \rightarrow+\infty$, then $f \in H^{p}$ for all $p$ satisfying $0<p<+\infty$.

We remark that while weakening the hypothesis of (1) by replacing the condition " $A(R)$ bounded" by the condition " $A(R)\left(R^{-2} \log R\right) \rightarrow 0$ as $R \rightarrow+\infty$," we have been able to strengthen the conclusion and get $f \in H^{p}$ for all $0<p<+\infty$. This new hypothesis is almost best possible since the inequality $A(R) R^{-2} \leq \pi$ holds for any complex-valued function $f$.

We remark further that the proof will make no use of the fact that the domain of $f$ is the unit disk, and hence the Theorem remains valid if.$J$ is replaced by any region.

Proof. Without loss of generality, we assume that $f(0)=0$ and that $f$ is unbounded. For $t>0$, we define

$\theta(t)=$ Angular Lebesgue measure of $[\{|z|=t\} \cap f(\Delta)]$,

$\alpha(t)=$ Angular Lebesgue measure of longest subarc of $[\{|z|=t\} \cap f(\Delta)]$,

Received by the editors September 20, 1973.

AMS (MOS) subject classifications (1970). Primary 30 A78.

Key words and phrases. Hardy classes, functions with finite area. 
and

$$
\begin{aligned}
\chi(t)=0 & \text { if }\{|z|=t\} \subset f(\Delta), \\
& =1 \quad \text { if }\{|z|=t\} \not \subset f(\Delta) .
\end{aligned}
$$

For $R>1$, let

$$
B(R)=\frac{\pi}{\log R} \int_{1}^{R} \frac{\chi^{(t)}}{t \alpha(t)} d t
$$

Theorem 3.1 of [2] states that $f \in H^{p}$ for all $p$ satisfying $0<p<$ $\lim$ inf $_{R \rightarrow+\infty} B(R)$, and thus our theorem will follow if we can show that $B(R) \rightarrow+\infty$ as $R \rightarrow+\infty$. From the definitions of $\theta$ and $a$ we get the inequalities

$$
\begin{aligned}
A(R) & =\int_{0}^{R} t \theta(t) d t \geq \int_{0}^{R} t \alpha(t) d t \\
& \geq \int_{[0, R] \cap\{a(t)=2 \pi\}} t \alpha(t) d t=2 \pi \int_{[0, R] \cap\{a(t)=2 \pi\}} t d t .
\end{aligned}
$$

Let $m(R)=$ Lebesgue measure of $[0, R] \cap\{\alpha(t)=2 \pi\}$. Then, since $g(t)=t$ is an increasing function of $t$, we get

$$
A(R) \geq 2 \pi \int_{0}^{m(R)} t d t=\pi[m(R)]^{2} .
$$

Multiplying both sides of this inequality by $R^{-2} \log R$ and recalling the original assumption on $A(R)$, we conclude that $R^{-1} m(R) \rightarrow 0$ as $R \rightarrow+\infty$.

Now $[R-m(R)] \leq \int_{0}^{R} \chi(t) d t$, and hence

$$
[R-1-m(R)]^{2} \leq\left(\int_{1}^{R} \chi(t) d t\right)^{2}
$$

if $R-1-m(R) \geq 0$. An application of the Schwarz inequality yields

$$
\left(\int_{1}^{R} \chi(t) d t\right)^{2} \leq\left(\int_{1}^{R} t a(t) d t\right)\left(\int_{1}^{R} \frac{\chi^{(t)}}{t \alpha(t)} d t\right) \leq A(R)(1 / \pi) B(R) \log R .
$$

Since $R-1-m(R) \geq 0$ for large $R$, we get the inequality

$$
\pi[R-1-m(R)]^{2} / A(R) \log R \leq B(R) .
$$

Using the fact that $R^{-1} m(R) \rightarrow 0$ and $A(R) R^{-2} \log R \rightarrow 0$ as $R \rightarrow+\infty$, we conclude that $B(R) \rightarrow+\infty$ as $R \rightarrow+\infty$, which completes the proof.

\section{REFERENCES}

1. H. Alexander, B. A. Taylor and J. L. Ullman, Areas of projections of analytic sets, Invent. Math. 16 (1972), 335-341. MR 46 \#2078.

2. Lowell J. Hansen, Hardy classes and ranges of functions, Michigan Math. J. 17 (1970), 235-248. MR $41 \# 7118$.

DEPARTMENT OF MATHEMATICS, WAYNE STATE UNIVERSITY, DETROIT, MICHIGAN 48202 